\title{
Diabetic retinopathy and carbohydrate metabolism
}

\section{By Yvonne C. Solomon*, Northgate Hospital, Great Yarmouth}

Those of you who have had dealings with diabetics will be only too aware of the disabling nature of diabetic retinopathy. This condition is a long-term complication of diabetes that often does not become manifest for 10-20 years after the onset of the disease. Initially the sufferer may experience visual disturbance, but as the condition progresses the result is total blindness. Diabetic retinopathy is now the most common cause of non-congenital blindness in this country.

The pathology is well described by many workers in the field (Caird \& Pirie, 1969; Kohner \& Oakley, 1975). Essentially diabetic retinopathy is a disease of the microvascular system in the retina. Blood is supplied by the optic artery and enters the retina near the optic disc. From here arteries radiate out and branch to form a capillary network lying within the retina. Initially microaneurysms, areas of focal thickening and capillary non-perfusion are seen. Eventually these progress to exudates, haemorrhages and the proliferative stages of new vessel formation. Although sufferers from this condition have been helped considerably in the last few years with the techniques of photocoagulation, these do not necessarily offer a permanent cure or provide a method of prevention. In order to have any hope of permanent prevention much more needs to be understood about the initial pathological lesion occurring in the retinal vascular system.

Epidemiological studies have not provided us with any strong lead, although it is well established that the risk of retinopathy increases with the duration and the age at onset of the diabetes (Sorsby, 1966). Increasingly, though, investigators and clinicians in this field are becoming convinced of the importance of well-controlled, low blood glucose concentrations, in the prevention of the onset of long-term diabetic complications (Cahill et al. 1976).

Retinopathy is only one of a number of long-term complications of diabetes. Others include cataract, nephropathy and neuropathy. It is of interest that the tissues affected (retina, lens, kidney and nervous tissue) all share the characteristic of being insensitive to the effects of insulin.

Tissues such as muscle and adipose tissue are sensitive to the effects of insulin. In these tissues insulin allows glucose carried in the blood to be taken up. These insulin-sensitive tissues never exhibit any of the long-term degenerative changes of diabetes, whereas the tissues affected by the long-term complications of diabetes are all insensitive to insulin. This means that they bear the full brunt of any raised blood glucose levels. As the blood glucose rises the level of glucose in these tissues also rises. The 'juvenile-onset' type of diabetes is of course characterized by a lack of endogenous insulin where as the 'maturity-onset' diabetics often exhibit hyper-

-Previous papers written as Y. C. Hamlett.

$0029-6651 / 79 / 3833-3106801.00$ C) 1979 The Nutrition Society. 
insulinaemia. This suggests that the long-term complications of diabetes are more likely to result from long-term hyperglycaemia than any lack of insulin.

That so little is known of the underlying biochemical pathology of diabetic retinopathy is to some extent explained by the nature of the tissue. Live human retinal tissue cannot be biopsied. Thus to investigate the disease fully an animal model is required. It was during the search for a suitable animal model that a relationship was found between retinopathy and the nature of the carbohydrate in the diet.

To produce a good animal model of diabetic retinopathy is problematic. The clinical changes of human diabetic retinopathy do not generally become apparent for maybe 10-20 years after the onset of the diabetes. The lifespan of most animals is short and were they to live long enough to produce a retinopathy, they would be unsuitable for research. However, if you induce diabetes in a rat by the intravenous injection of streptozotocin (a drug which appears to selectively 'knock out' the $\beta$ cells in the islets of Langerhans in the pancreas) then a retinopathy is produced in the rat whose characteristics are shared by the initial stages of human retinopathy.

Preparations of retinal vascular systems in the diabetic rat show morphological changes after 6 months of diabetes.

Cohen and his associates (Cohen et al. 1972) found that normal (not diabetic) rats maintained on diets in which the carbohydrate content of the diet was provided by sucrose developed a retinopathy similar to diabetic retinopathy. My colleagues and I repeated these experiments using four groups of rats (Papachristodoulou et al. 1976). Two groups were rendered diabetic and two remained normal.

One diabetic and one normal group were given the carbohydrate in their diet as sucrose $(680 \mathrm{~g} / \mathrm{kg})$ and the other normal and diabetic groups were given starch as the source of carbohydrate in the diet. The rats were killed after 6, 9 and 12 months and histological preparations were made of their retinal vascular systems. The extent of the retinopathy seen in the sucrose-fed normal rat was identical to that found in the starch-fed diabetic group. When the sucrose feeding was superimposed on the diabetic state then the changes were even more pronounced. No changes were seen in the starch-fed normal retinas.

Why should a diet high in sucrose produce a retinopathy in normal rats identical to that produced in the streptozotocin-diabetic rats?

Sucrose on ingestion is broken down into its component parts, glucose and fructose, and the sugar is absorbed in this simplified form. In order to identify which dietary sugar was responsible for these changes the experiment was repeated with diets containing various sources of carbohydrate (Boot-Handford \& Heath, 1977).

Six groups of rats were used and these were all given a series of highcarbohydrate diets ranging from those containing unrefined cereals (MRC $4 \mathrm{IB}$; wheat and oats) to diets constituted using highly-refined sugars (glucose, fructose and sucrose). A diet containing cereal and fructose (MRC $4 \mathrm{IB} / \mathrm{FR}$ ) was also tested. These diets were fed for 6 months $a d$ lib. and the extent of retinopathy was 
assessed in histological preparations of the rat retinal vascular systems. The results are shown in Table 1 . From this it can be seen that fructose is identified as the dietary constituent responsible for the retinal changes.

Table 1. Changes in retinal vascular systems after 6 months feeding on specified diet

Diets

Degree of retinopathy

No retinopathy
Very mild retinopathy
Mild retinopathy
Moderate retinopathy

Starch MRC 4 IB Glucose Fructose Sucrose $\mathrm{MRC}_{4} \mathrm{IB} / \mathrm{FR}$

This leads us to the question; how can a diet with a high fructose content cause a retinopathy in normal rats identical in character to that produced by hyperglycaemia in the diabetic rat?

A lead was presented by the activity of the sorbitol pathway. At present a possible mechanism for the pathogenesis of diabetic cataract was being elucidated. This involves the sorbitol pathway (Fig. I). This pathway has been little investigated until recently. The pathway involves only two steps. First the conversion of glucose directly to sorbitol and second the conversion of sorbitol to fructose.

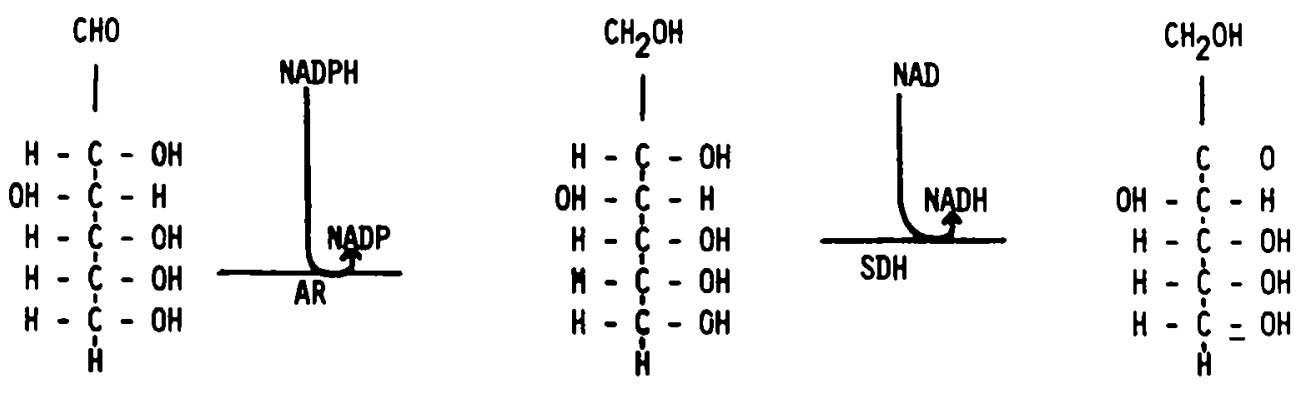

GLUCOSE

SORBITOL

FRUCTOSE

Fig. I. Schematic diagram representing the sorbitol pathway.

The first reaction step is mediated by the enzyme aldose reductase ( $E C$ I.I.I.2 I). This enzyme has a high Michaelis constant which means that glucose is only converted to sorbitol when glucose is present in high concentrations. This pathway has then particular importance in the diabetic, in insulin-insensitive tissues which are exposed to the high level of blood glucose. In the lens of the diabetic, glucose carried there by the blood, readily enters the tissue and the high concentration allows sorbitol to be produced in large amounts. The second step in the sorbitol pathway is the conversion of sorbitol to fructose by the enzyme sorbitol dehydrogenase (L-iditol dehydrogenase, $E C$ r.1.1.14). In the lens sorbitol dehydrogenase is present in only small amounts or is inactive. This causes a partial block in the pathway and allows sorbitol to build up to osmotically damaging levels. 
Water is drawn into the lens to restore the osmotic balance, but eventually permanent damage is caused as the lens fibres burst and the lens becomes opaque (Kinoshita, 1974). This is a simplified explanation of the mechanism involved in the pathogenesis of diabetic cataract.

The question therefore arose as to whether this or some other similar mechanism could be acting in the retina?

Might the hyperglycaemia in the diabetic rats cause high levels of glucose in the retina and thus the subsequent production of sorbitol, whereas in the normal rat, fructose from dietary sources might be taken up by the retina and converted to sorbitol by means of the enzyme sorbitol dehydrogenase. Although most fructose is removed by the liver from the portal blood supply, some fructose does enter the systemic circulation when rats are given a high-fructose diet. This pathway then gives us the potential of a connexion between diabetes, hyperglycaemia and fructose.

In this experiment, four groups of rats were taken; two were rendered diabetic with streptozotocin and two remained normal. One of each pair was given a highsucrose diet while the other received the carbohydrate as starch. After 3 weeks the animals were killed and their retinas analysed for glucose, fructose and sorbitol (Heath \& Hamlett, 1976). We found that the sorbitol pathway was active in the retina and that sorbitol accumulated in the diabetic retina.

However, the levels of sorbitol were not large enough to cause osmotic damage and no increase in sorbitol was found in the fructose-fed normal retinas (Fig. 2).

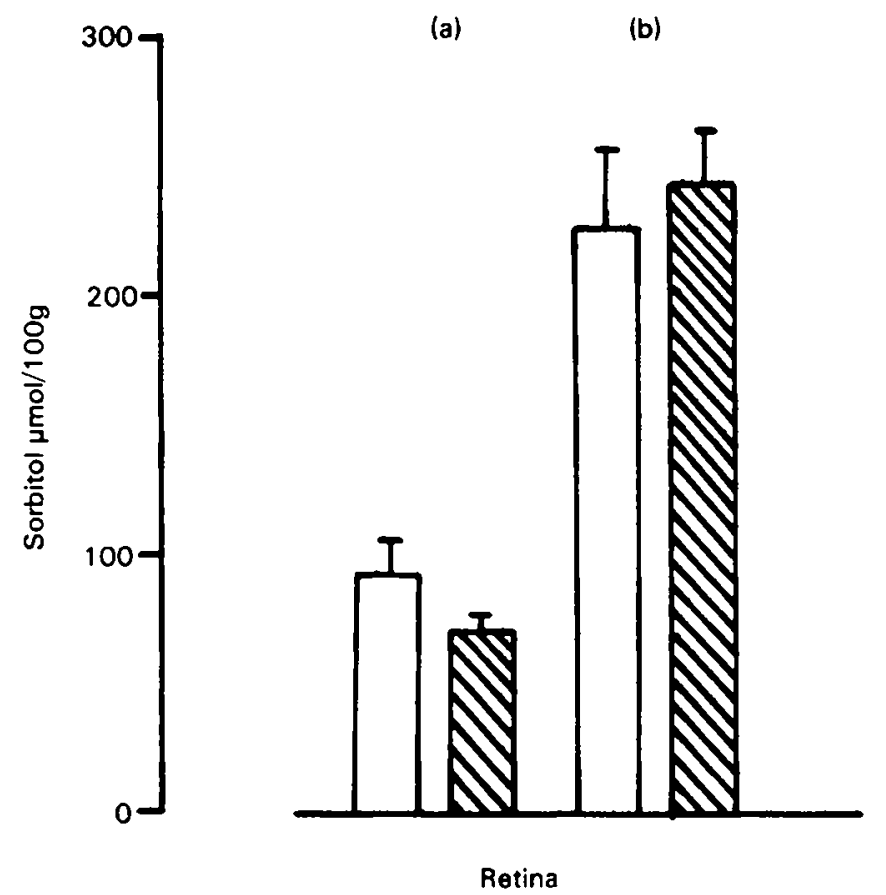

Fig. 2. Levels of sorbitol in the retinas of (a) normal and (b) diabetic rats fed on ( $\square$ ) sucrose and () starch diets. 




Fig. 3. Levels of fructose in the retinas of (a) normal and (b) diabetic rats fed on ( $\square$ ) sucrose and (D) starch diets.

(a) (b)
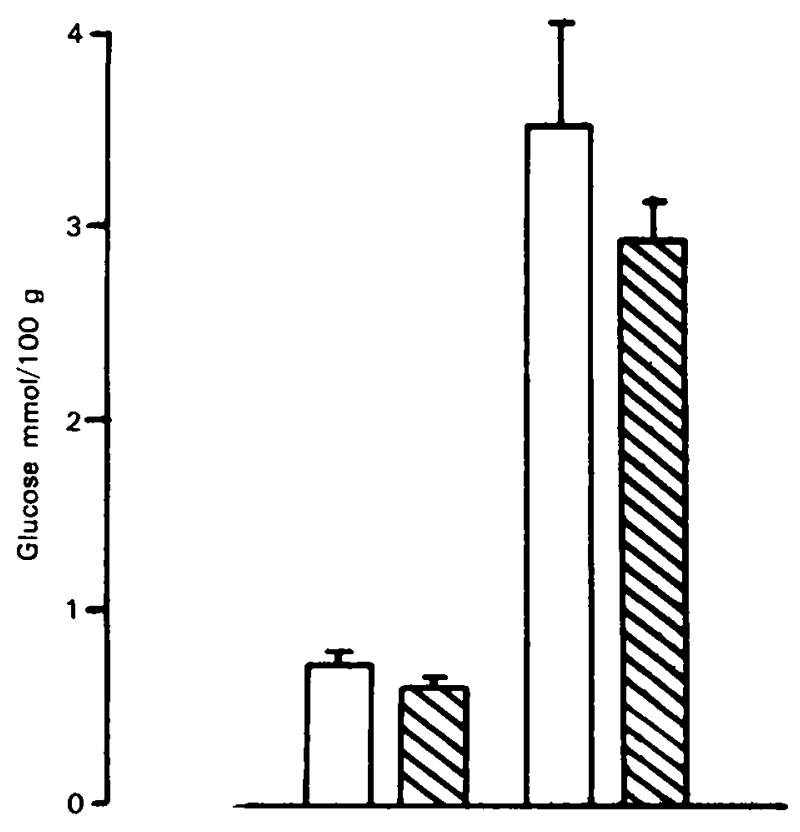

Retina

Fig. 4. Levels of glucose in the retinas of (a) normal and (b) diabetic rats fed on (D) sucrose and (口) starch diets. 
This indicated that whatever the retinal toxin was, sorbitol did not look a strong contender, and that the mechanism operating in the pathogenesis of diabetic cataract was not responsible for the changes seen in retinopathy. This was perhaps explained by the activity of the enzyme sorbitol dehydrogenase in the retina which converted the sorbitol to fructose. As can be seen in Fig. 3 fructose levels were significantly raised in the diabetic retinas. Other tissues such as blood and liver did not show similar levels or increases in the diabetics, thus supporting the belief that the retinal sorbitol and fructose were endogenous to the retina in origin. Indeed a good correlation between sorbitol and fructose levels in the retina was found. Glucose levels in the retina were similar to those found in the blood (Fig. 4).

We now had before us a mechanism by which glucose in the hyperglycaemic animal could be metabolized other than by the better-known Embden Meyerhoff and pentose phosphate pathways (the end product of this pathway being fructose-. the very sugar that seemed to be producing retinopathy in the non-diabetic animal when fed as a dietary constituent).

Fig. 5 demonstrates the normal glycolytic pathway for glucose (the Embden Meyerhoff pathway) and the alternate pentose phosphate and sorbitol pathways. If glucose is metabolized by the sorbitol pathway then fructose is the end product. It was difficult to see how fructose produced by this pathway could alter the metabolism of the retina, since apart from in the liver, it was generally accepted that fructose is phosphorylated to fructose-6-phosphate (F6P). F6P is itself a normal glycolytic intermediate and further metabolism of fructose would be the same as for glucose which had been metabolized directly through the Embden Meyerhoff pathway.

In liver there is an alternative enzyme for the phosphorylation of fructose; a specific fructokinase and fructose-I-phosphate $\left(F_{I} P\right)$ is formed. $F_{I} P$ is cleaved to form one molecule of dihydroxyacetone phosphate (DHAP) and one molecule of glyceraldehyde (GA). By contrast F6P is further phosphorylated to fructose-1,6diphosphate (F I6-diP) before being cleaved to form one molecule of glyceraldhyde phosphate (GAP) and one of DHAP.

One of the most important controlling steps in glycolysis is that operating on the conversion of F6P to $F_{1} 6$-diP since this reaction is not reversible. Were glucose to be metabolized by the sorbitol pathway to fructose and thence to $F_{I} P$ the normal glycolytic controls would be bypassed.

Another experiment was set up to investigate the route by which fructose is metabolized in the diabetic retina. One group of rats was made diabetic by the intravenous injection of streptozotocin and another group served as a control. After $24 \mathrm{~d}$ their retinas were analysed for levels of F I P, F6P and G6P (Hamlett \& Heath, 1977). The results are shown in Fig. 6.

These results indicate the presence of a specific fructokinase in the retina for the phosphorylation of fructose to $F_{I} P$. The level of $F_{I} P$ in the diabetic retina showed a ten-fold increase over that in the normal retinas. By contrast the levels of F6P were not elevated suggesting that fructose is metabolized preferentially by this fructokinase in the retina. This metabolic route bypasses the normal glycolytic 


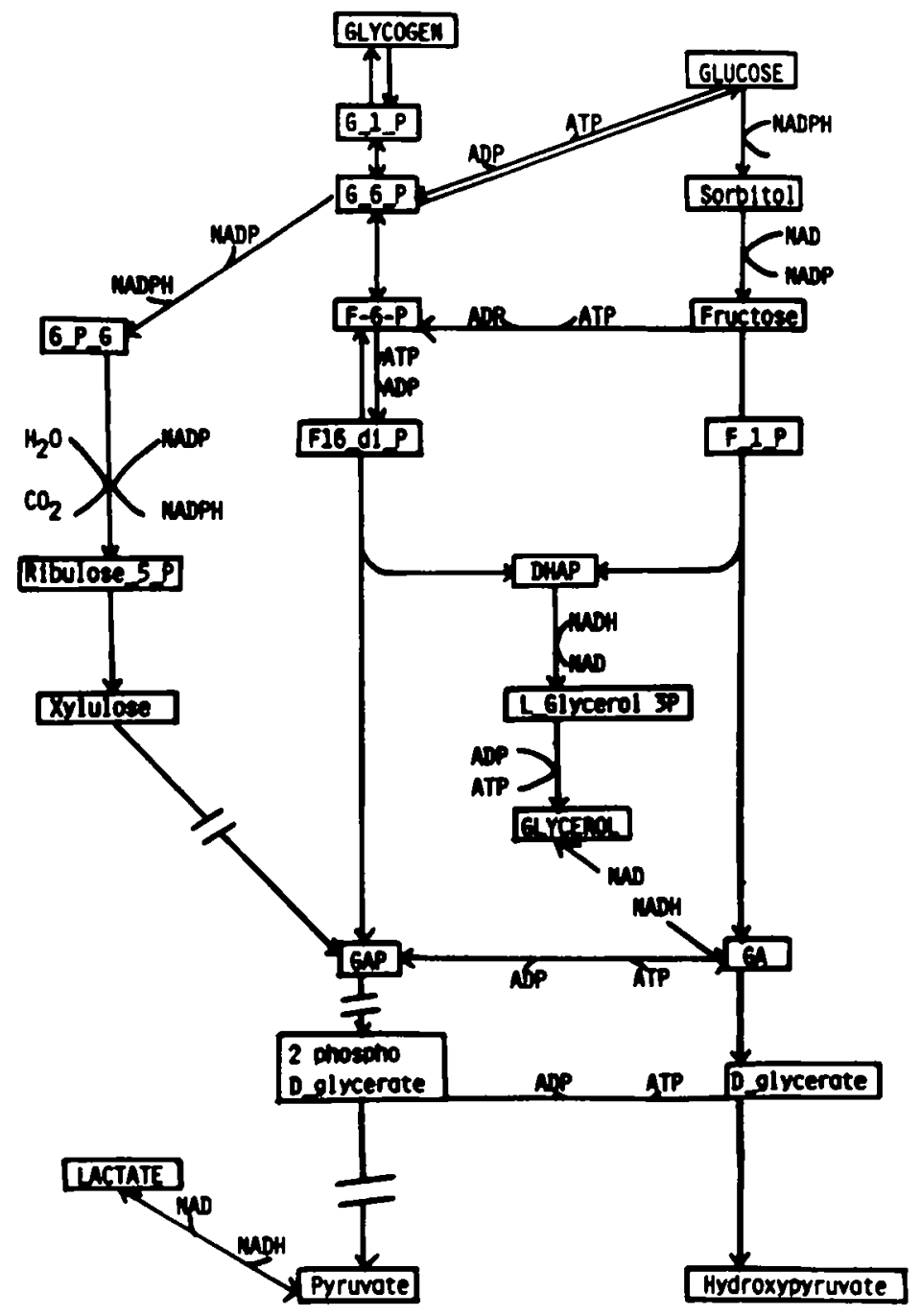

Fig. 5. The sorbitol pathway in relation to glucose by the Embden Meyerhoff and pentose phosphate pathways.

controls operative at the phosphofructokinase step of glycolysis and indicates a way by which uncontrolled glucose metabolism may operate in the diabetic retina.

How this may eventually lead to retinal damage is as yet unclear. However, the end product of this metabolic route is lactate. Increased levels of lactic acid have been found in the diabetic rat retina (Heath et al. 1975). Lactate formation has also been implicated as a pathogenic agent in the development of retinopathy by a number of workers (Kohner \& Oakley, 1975; Papachristodoulou, 1976). Future research may show us if there is any foundation to these hypotheses.

What though of the diabetic patient today? The dietary use of fructose and sorbitol is advocated for many diabetics since they can provide energy independently of insulin and do not immediately lead to an increase in blood 


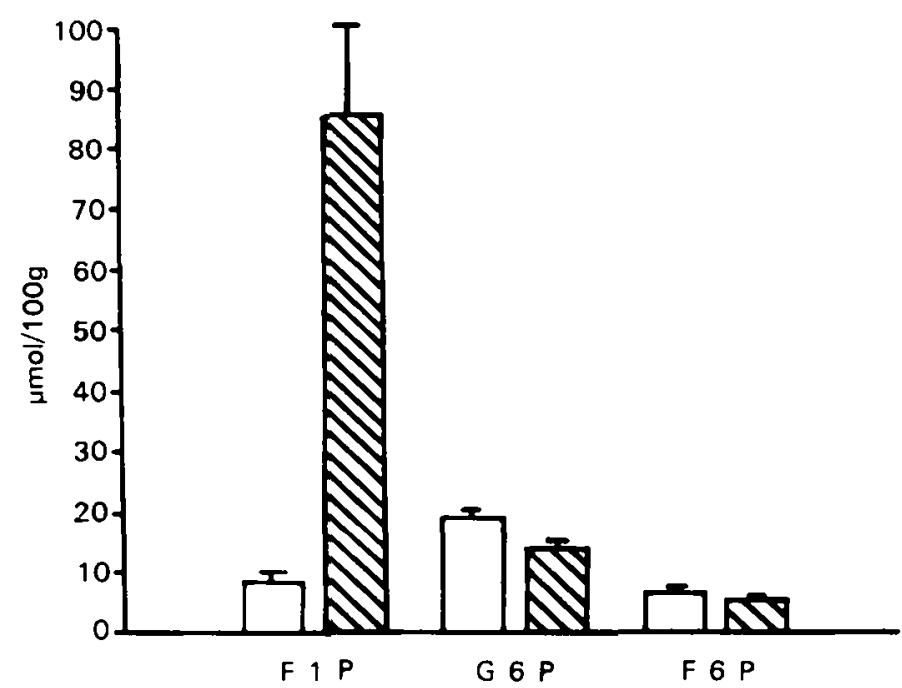

Fig. 6. Retinal levels of fructose-1-phosphate $\left(F_{I} P\right)$, glucose-6-phosphate $(G 6 P)$ and fructose6-phosphate (F6P) in ( $\square$; eight rats) normal and ( $\square$; nine rats) diabetic rats.

glucose levels. Although it must be borne in mind that both yield trioses which can be converted to glucose in the liver. Are we perhaps advocating exactly the wrong sugar substitutes for diabetics? Sorbitol and fructose do provide sweetness and energy to the diabetic without rapid hyperglycaemia and caution should be exercized before we radically change our policy for diabetics bearing in mind that these results are based on rats given diets containing at least $340 \mathrm{~g}$ fructose $/ \mathrm{kg}$. It would seem better to try and achieve good diabetic control wherever possible. Let us hope that research will provide us with some of the answers in the near future so that the disabling effects of diabetic retinopathy can be prevented.

\section{REFERENCES}

Boot-Handford, R. P. \& Heath, H. (1977). I.R.C.S. Med. Sci. 5, 583 .

Cahill, G. F. Jr, Etzwiler, O. D. \& Frienkel, N. (1976). Diabetes 25, 237.

Caird, F. L. \& Pirie, A. (eds.) (1969). Diabetics and the Eye, pp. I and I1. Oxford and Edinburgh: Blackwell Scientific Publications.

Cohen, A. M., Michaelson, L. C. \& Yanko, L. (1972). Am. F. Ophthal. 73, 863.

Hamlett, Y. C. \& Heath, H. (1977). I.R.C.S. Med. Sci. 5, 510.

Heath, H. \& Hamlett, Y. C. (1976). Diabetologia I2, 43.

Heath, H., Kang, S. S. \& Philippou, D. (1975). Diabetologia I1, 57.

Kinoshita, H. H. (1974). Invest. Ophthal. 13, 713.

Kohner, E. M. \& Oakley, N. W. (1975). Metabolism 24, 1085.

Papachristodoulou, D. (1976). Development of retinopathy in sucrose-fed and streptozotocin diabetic rats. PhD Thesis, University of London.

Papachristodoulou, D., Heath, H. \& Kang, S. S. (1976). Diabetologia 12, 367.

Sorsby, A. (1966). Rep. publ. Hlth med. Subj. no. 114 .

\section{Printed in Great Britain}

